Classification

Physics Abstracts

$73.20-73.90-72.20 \mathrm{~J}-78.55 \mathrm{D}$

\title{
Surface recombination, surface states and Fermi level pinning
}

\author{
J. M. Moison and M. Bensoussan \\ Laboratoire de Bagneux, Centre National d'Etudes des Télécommunications, 196, avenue H.-Ravéra, \\ F-92220 Bagneux, France
}

(Reçu le 21 octobre 1986, accepté le 9 janvier 1987)

\begin{abstract}
Résumé. - Les processus de recombinaison aux surfaces et aux interfaces, dont l'importance croît avec l'apparition de dispositifs optoélectroniques de petite taille, ne sont pas encore bien compris, à cause du faible nombre de données expérimentales disponibles. On présente ici les premières mesures simultanées de la densité et de la position des états de surface, de la position du niveau de Fermi à la surface et de la vitesse de recombinaison de surface, pour divers traitements de la surface de InP. On montre que les modèles classiques décrivent correctement les relations entre ces quantités. On décrit une méthode pour moduler (réduire) la vitesse de recombinaison de surface en agissant sur les états de surface, avec des exemples d'applications à d'autres semiconducteurs comme Si ou GaAs.
\end{abstract}

\begin{abstract}
Surface and interface recombination processes, which are becoming more and more important with the appearance of small-size optoelectronic devices, are still not well understood because reliable data are very scarce. We report here the first simultaneous in situ measurements of the density and position of surface states, of the position of the Fermi level at the surface, and of the surface recombination velocity, for various treatments of the surface of InP. Classical models are shown to describe correctly the relations between those quantities. A method for engineering (reducing) the surface recombination velocity in various situations by acting on the surface states is outlined, and applications to other semiconductors such as $\mathrm{Si}$ and GaAs are presented.
\end{abstract}

\section{Introduction.}

Surfaces and interfaces of semiconductor materials are usually places where the atomic positions and therefore the local electronic structure are highly perturbed with respect to the adjacent bulk(s). The corresponding localized states may act as carrier recombination channels, localized as well. When excess carriers are continuously injected in the material, their stationary density is smaller near the interface than in the bulk because of their reduced lifetime. The depleted zone actually extends far inside the bulk, up to microns away from the interface, since carriers drift from the bulk to the interface to reduce the density gradient. The depletion effect in turn decreases whatever effect is expected from the carrier injection near the interface. For instance, the yield of light detectors and emitters is reduced by interface recombination, respectively by loss of photogenerated e-h pairs and by non-radiative recombination of junction-injected pairs. It is therefore important to try to control the interface recombination processes and hence to study them. In the following, we shall take the surface as an example of interface, but all concepts developed in this case may be applied to any interface as well.

The efficiency of surface recombination processes is expressed by the surface recombination velocity $V_{\mathrm{sr}}$, which may be understood as the ratio of the «thickness of the surface" to the "lifetime at the surface ", both ill-defined quantities. The simplest theoretical evaluation of $V_{\text {sr }}$, developed by Stevenson and Keyes on the basis of the bulk-dedicated Shockley-Read-Hall formalism $[1,2]$ yields :

$$
V_{\mathrm{sr}}=N_{\mathrm{t}} \frac{S_{\mathrm{n}} S_{\mathrm{p}} V_{\mathrm{n}} V_{\mathrm{p}}\left(n_{\mathrm{b}}+p_{\mathrm{b}}\right)}{S_{\mathrm{n}} V_{\mathrm{n}}\left(n_{\mathrm{s}}+n_{1}\right)+S_{\mathrm{p}} V_{\mathrm{p}}\left(p_{\mathrm{s}}+p_{1}\right)}
$$

where $n_{\mathrm{s}}$ and $p_{\mathrm{s}}$ are the electron and hole densities (per unit area) in the $N_{\mathrm{t}}$ surface states lying at an energy $E_{\mathrm{t}}$ under injection conditions, $n_{1}$ and $p_{1}$ the densities in this state when the Fermi level $E_{\mathrm{f}}$ coincides with $E_{\mathrm{t}}$ and $S_{\mathrm{n}}, S_{\mathrm{p}}, V_{\mathrm{n}}$ and $V_{\mathrm{p}}$ the capture cross-sections and thermal velocities. The scale of $V_{\mathrm{sr}}$ is then set by the thermal velocity and it depends on the density of the surface states, on their capture cross-sections, and also on the way they are filled, i.e. at near-equilibrium on the position of the Fermi 
level at the surface. In order to check the validity of equation (1), all these quantities should be independently measured and their respective weight evaluated. This could not be done up to now for two main reasons. The first one is that measuring $V_{\text {sr }}$ is not by far an easy task. As we shall show in more detail below, it cannot usually be obtained directly, since the exchange of carriers between bulk and surface is unavoidable, but through measurements which involve complicated transport processes, perturb the sample and bias the value of $V_{\mathrm{sr}}$ itself. The second reason is that the characterization and control of the surfaces, and to a much larger extent of the interfaces involved, is quite complex and was nearly out of reach until 1970 . Finally, even though surface recombination has been recognized and modelized as soon as 1950, the lack of sound data obtained on well-characterized systems prevented from going much beyond the phenomenological description of the observations. In a recent review of the state of the art of recombination at surfaces and interfaces [3], Aspnes concludes that the available data are consistent with the assumption that the surface recombination is mainly governed by changes of the Fermi level and that mid-gap surface states play the leading part, though alternative explanations are possible in view of the lack of reliable data. We report here new extended data in the case of the InP surface which rather indicate that, in most usual cases, near-band-edge gap surface states govern the surface recombination velocity.

\section{The surface of (100)InP : a model system ?}

If we look for a model system for testing the theoretical models, it is clear that at present it is far easier to determine directly at least the electronic states and the position of the Fermi level at a surface than at an interface. If we add that InP has a surface recombination velocity which may be changed by surface treatments [4], its surface is a priori an excellent candidate for our purpose. Indeed, in a recent paper [5], we have reported what we think is the first in situ determination of most parameters involved, i.e. the surface recombination velocity, the density of surface states and the position of the Fermi level at the InP surface. The surface treatments studied include cleavage under vacuum for (110) surfaces, chemical polishing, cleaning under vacuum by ion etching and annealing, annealing under an arsenic pressure, and oxygen adsorption on the clean surface for (100) surfaces. For the various surfaces obtained, the surface recombination velocity is deduced from in situ photoluminescence yield measurements [5], the energy distribution of occupied surface states from ultraviolet photoemission spectroscopy, and the position of the lowest empty surface band from electron-energy-loss spectroscopy [6].
Those surface techniques are established ones and their results need not be discussed here, since the interpretation is rather straightforward. On the other hand, it must be emphasized that the measurement of the surface recombination velocity is by no means a straightforward affair. Most techniques and ours - are based on the determination of the influence of the surface on bulk excess carrier densities or on their decay time [4 and Ref. therein]. Since bulk-surface carrier exchanges are then required and anyway unavoidable, the extraction of $V_{\text {sr }}$ from 'raw data (photon- or electron-excited luminescence yield, decay time of carrier gradients,...) requires a sound knowledge of the transport parameters (bulk recombination lifetime, diffusion coefficient, ...) and of the system (boundary conditions, electric field, ...). The measurement itself may alter those quantities, for instance when intense carrier generation decreases the built-in electric field (band flattening). It may also bias the value of $V_{\text {sr }}$ itself, since $V_{\text {sr }}$ depends on the Fermi level in the band gap at the surface, which may be moved by band flattening. Conversely, the carrier density near the surface which influences the band bending depends on the surface recombination velocity, since it results from a balance between surface and bulk recombination, generation and diffusion. From these examples, it is clear that in a general case, and especially when the injected carrier density is larger than the minority carrier concentration which is nearly always - , surface recombination data should be extracted from a complete calculation involving all the perturbations of the system which is seldom the case.

Our own approach was simplified by the strong pinning of the Fermi level observed on (100)InP : whatever the surface treatment and the doping level from $\mathrm{n} .10^{18} \mathrm{~cm}^{-3}$ to $\mathrm{p} .10^{18} \mathrm{~cm}^{-3}$, the Fermi level stays always located at $1.0 \pm 0.1 \mathrm{eV}$ above the valence band maximum. This implies the existence of about $10^{12} \mathrm{~cm}^{-2}$ surface states located around midgap [6]. A consequence of this strong pinning is the stability of the space-charge layer under illumination even when a high carrier density (up to $10^{16} \mathrm{~cm}^{-3}$ ) is generated (negligible photovoltage effect). The «dead layer» involved in luminescence yield measurements is then stable and the quasi-Fermi level of majority carriers stays unmoved. On the other hand, the quasi-Fermi level of minority carriers moves considerably in usual conditions of photoluminescence measurements : for a $10 \mathrm{~W} / \mathrm{cm}^{2}$ illumination, the injection level just outside the space-charge layer is $10^{14} \mathrm{~cm}^{-3}$ and for a n-type material the hole quasi-Fermi level drops down by $1 \mathrm{eV}$. Since it usually can be assumed that the quasi-Fermi levels are flat across the space-charge layer, the filling of the surface states and in turn the efficiency of surface recombination are modified; this is also true under 
usual conditions of device operation. Actually, the unpinning of the quasi-Fermi levels and the surface recombination change are simultaneous and stem from similar causes since the position of the Fermi level is dictated by a balance between generation and recombination.

More precisely, under quasi-equilibrium conditions where the excess carriers are less than the minority carriers, $V_{\mathrm{sr}}$ may be expressed in the Stevenson-Keyes model as follows :

$$
\begin{aligned}
V_{\mathrm{sr}} & =N_{\mathrm{t}} \frac{n_{\mathrm{b}}+p_{\mathrm{b}}}{2 n_{\mathrm{i}}} \times \\
& \times \frac{S V_{\mathrm{th}}}{\cosh \left[\left(E_{\mathrm{t}}-E_{\mathrm{i}}\right) / k T\right]+\cosh \left[\left(E_{\mathrm{f}}-E_{\mathrm{i}}\right) / k T\right]}
\end{aligned}
$$

where $N_{\mathrm{t}}$ is the density of traps at energy $E_{\mathrm{t}}$ and $S$ their ambivalent capture cross-section, $V_{\text {th }}$ the thermal velocity, $n_{\mathrm{i}}, n_{\mathrm{b}}$ and $p_{\mathrm{b}}$ the bulk intrinsic and mobile carrier concentrations, and $E_{\mathrm{f}}$ and $E_{\mathrm{i}}$ the Fermi and intrinsic levels at the surface. This equation shows that all else being equal the traps for which $\left|E_{\mathrm{t}}-E_{\mathrm{i}}\right|<\left|E_{\mathrm{f}}-E_{\mathrm{i}}\right|$, i.e. located nearer midgap than the Fermi level are considerably more efficient than the other ones. For InP in the lowinjection regime, a small band of surface states ( $0.6 \mathrm{eV}$-wide centered at midgap) governs the surface recombination. On the other hand, under more current situations such as ours where excess carriers are more numerous than minority carriers but less numerous than majority carriers, $V_{\mathrm{sr}}$ may be expressed within the same model by (3):

$$
V_{\mathrm{sr}}=N_{\mathrm{t}} \frac{n_{\mathrm{b}}+p_{\mathrm{b}}}{2 n_{\mathrm{i}}} \times \frac{S V_{\mathrm{th}}}{\cosh \left[\left(E_{\mathrm{t}}-E_{\mathrm{i}}\right) / k T\right]+\exp \left[\left(F_{\mathrm{n}}-F_{\mathrm{p}}\right) / k T\right] \cosh \left[\left(F_{\mathrm{n}}+F_{\mathrm{p}}-2 E_{\mathrm{i}}\right) / 2 k T\right]}
$$

where $F_{\mathrm{n}}$ and $F_{\mathrm{p}}$ are the electron and hole quasiFermi levels.

At a moderate injection level of $10^{14} \mathrm{~cm}^{-3}$, $F_{\mathrm{n}}-F_{\mathrm{p}}$ is $1.0 \mathrm{eV}$ and $\left(F_{\mathrm{n}}+F_{\mathrm{p}}-2 E_{\mathrm{i}}\right)$ is $-0.2 \mathrm{eV}$, so that the second term in the denominator of (3) is much larger than the first one for all trap energies lying in the band gap. Hence all surface gap states contribute equally to the recombination process.

This is an important change since the distribution of surface states in the band gap is far from uniform. It is rather U-shaped, with a minimum near midgap of $10^{12} \mathrm{~cm}^{-2}$ states which are responsible for the pinning of the Fermi level, and maxima near the band edges of $10^{14} \mathrm{~cm}^{-2}$ states which can be detected respectively by UPS and EELS. Therefore, at high

Table I. - Position of the Fermi level at the surface with respect to the top of the valence band $\left(E_{\mathrm{f}}-E_{\mathrm{v}}\right)$, surface recombination velocity $V_{\mathrm{s}}$, density of nearvalence-band gap surface states $N_{\mathrm{sv}}$ detected by UV photoemission and of near-conduction-band gap surface states $N_{\mathrm{sc}}$ detected by electron-energy-loss spectroscopy, for various treatments of the n-type (100) InP surface (CP : chemical polishing; As : annealing under As pressure; Ox : $10^{4}$ Langmuir exposure of the clean surface to oxygen; $\mathrm{Cl}$ : cleaned and annealed surface; Am : surface amorphized by $3 \times 10^{13} \mathrm{~cm}^{-2}$ ion bombardment).

\begin{tabular}{|l|cccc|}
\hline Surface & $\begin{array}{c}V_{\mathrm{sr}} \\
\left(10^{5} \mathrm{~cm} / \mathrm{s}\right)\end{array}$ & $\begin{array}{c}E_{\mathrm{f}}-E_{\mathrm{v}} \\
(\mathrm{eV})\end{array}$ & $\begin{array}{c}N_{\mathrm{sv}} \\
\left(10^{14} \mathrm{~cm}^{-2}\right)\end{array}$ & $\begin{array}{c}N_{\mathrm{sc}} \\
(\text { a.u. })\end{array}$ \\
\hline$(\mathrm{CP})$ & 0.0 & 1.0 & $<0.1$ & $\ll 1$ \\
$(\mathrm{As})$ & 0.17 & 1.0 & 0.2 & $\ll 1$ \\
$(\mathrm{Ox})$ & 1.33 & 1.0 & 1.3 & $\ll 1$ \\
$(\mathrm{Cl})$ & 1.64 & 1.0 & 1.6 & 1 \\
$(\mathrm{Am})$ & 2.21 & 1.0 & 3.0 & $?$ \\
\hline
\end{tabular}

injection, the near-band-edge states play the first part because they are much more numerous. Since their density and the position of the quasi-Fermi levels are determined, the only unknown quantity in (3) is the capture cross-section $S$. The variation of $V_{\text {sr }}$ with filled and empty $N_{\mathrm{t}}$ obtained for various surface treatments (Table I and Fig. 1) confirms the validity of equation (3) and yields a $S$ value of $10^{-15} \mathrm{~cm}^{2}$, which indicates that surface states are attractive traps. It may be noted that the evaluation of our data with the commonly-used equation (2) yields a quite unrealistic value of $10^{-25} \mathrm{~cm}^{2}$. Finally, our results show that the Stevenson-Keyes formalism is indeed a good approach to the surface recombination velocity. We see now what are their implications on the engineering on this important parameter.

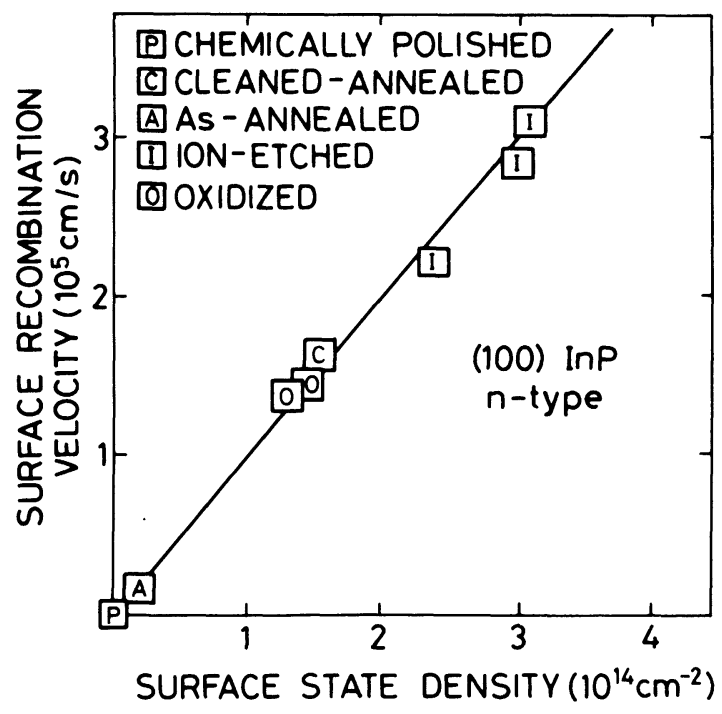

Fig. 1. - Surface recombination velocity $v s$. gap density of surface states near the valence band maximum for various treatments of n-type (100)InP. 
3. Engineering of the surface recombination velocity : reduction of the gap surface state density and/or Fermi level unpinning.

We consider now the problem of engineering - i.e. most often of reducing - the surface recombination for applied purposes. We start from the usual situation where the Fermi level at the surface is strongly pinned near midgap by intrinsic numerous surface states originating from the dangling bonds and the perturbed back bonds, and also most often from residual defects. In the low-injection regime (Eq. (2)), we must clearly try to reduce the density of surface states or shift the Fermi level towards either band edge. Actually, since the midgap states which govern $V_{\mathrm{sr}}$ are also those which pin the Fermi level, those two goals will be reached together. Let us assume that, for instance on a $10^{18} \mathrm{~cm}^{-3}$ n-type sample, we have succeeded in suppressing the band bending at the surface. This means that we have significantly reduced the midgap surface state density (below $10^{11} \mathrm{~cm}^{-2}$ ), the near-band-edge states being relatively unimportant. $V_{\mathrm{sr}}$ is then :

$$
V_{\mathrm{sr}}=\frac{N_{\mathrm{t}} n_{\mathrm{b}} S V_{\mathrm{th}}}{2 n_{\mathrm{i}}} \exp \left[-E_{\mathrm{g}} / 2 k T\right] \sim 10^{-9} N_{\mathrm{t}}
$$

Then in principle very low surface recombination velocities $(<100 \mathrm{~cm} / \mathrm{s})$ can be obtained this way. To get a feeling of such values, we may say that for a 1micron-thick InP sub-surface active region, surface recombination alone - in the absence of bulk recombination - would give a carrier decay time $\tau_{\mathrm{s}}$ in the microsecond range, while the bulk decay time $\tau_{b}$ lies in the nanosecond range, so that the surface recombination efficiency is negligible. On the other hand, for usual $V_{\text {sr }}$ values of $10^{5} \mathrm{~cm} / \mathrm{s}$, $\tau_{\mathrm{s}}$ lies in the nanosecond range and bulk and surface recombination efficiencies are comparable. The question is : can we reduce the density of nearmidgap surface states enough to unpin the Fermi level ? Actually, this is very difficult to achieve. Except for cleaved surfaces, most surfaces of IV elements or III-V compounds have their surface Fermi level pinned around midgap, and the same is probably true of ternary and quaternary compounds. At present, only well-matched interfaces such as $\mathrm{Si} / \mathrm{SiO}_{2}$ or $\mathrm{GaAs} / \mathrm{GaAlAs}$ have few enough gap states to free the Fermi level. However, chemical or photochemical treatments have been recently reported to reduce considerably the density of surface states, down to $10^{11} \mathrm{~cm}^{-2}$ for GaAs [7], and even $10^{7} \mathrm{~cm}^{-2}$ for Si [8], thus unpinning the Fermi level and lowering $V_{\mathrm{sr}}$ to a record value of $0.25 \mathrm{~cm} / \mathrm{s}$. The effect of such treatments is probably first to saturate most dangling bonds with oxygen and then to suppress the remaining states by hydrogen adsorption, just like at the $\mathrm{Si} / \mathrm{SiO}_{2}$ interface.

The situation is rather different at high injection levels. For a n-type sample, $F_{\mathrm{p}}$ reaches the valence band edge while $F_{\mathrm{n}}$ stays pinned near midgap. Equation (3) then also reduces to equation (4). We have here a dynamic unpinning of the quasi-Fermi level by injection, with the intrinsic

$$
N_{\mathrm{t}}\left(\sim 10^{14} \mathrm{~cm}^{-2}\right)
$$

surface states still present. Therefore, the $V_{\mathrm{sr}}$ values reach typically $10^{5} \mathrm{~cm} / \mathrm{s}$. Since all gap states contribute to $V_{\mathrm{sr}}$, we must now try to reduce not only the midgap density of states, but above all the much higher density of near-band-edge states. This is in a sense easier because those states can be directly observed by surface techniques, while the few midgap states remain invisible. Furthermore, we deal with states associated not with defects or other unpredictable origins, but merely from the broken bonds at the surface. The only action we may have on them, as long as a surface is involved, is to replace the intrinsic states lying in the band gap by others shifted away from it. A common way is oxygen adsorption, which forms a kind of bidimensional oxide with states located high inside the substrate bulk bands, thus reducing the gap density of states of $\mathrm{GaAs}$ [9] or InP $[6,10]$ and hence $V_{\text {sr }}$ (see Fig. 1 and Table I). Here again, liquidphase oxidation reduces considerably more the gap density of states, probably due to additional hydrogenation (see our data on the chemically-polished surface). A similar trick can be performed by placing on the sample an ultra-thin layer of an other material whose surface states are farther away from the band gap of the substrate than its intrinsic ones. This is the case of InP annealed under arsenic pressure, where a two-monolayer overlayer of InAs forms, giving to InP InAs-like surface states, located farther away from its band gap than its intrinsic ones [11]. This works both sides, since the deposition of one monolayer of InAs on GaAs gives it InAs-like surface states which are here nearer to its band gap than its own surface states, so that the surface recombination increases [12]. The use of such «artificial» surfaces still needs further experimental confirmation in other systems and a theoretical basis ; it has also probably limits since surface states originating from defects cannot be treated this way. Nevertheless, it yields a first methodology for the engineering of the surface recombination velocity on the basis of the knowledge gathered in surface physics. Its extension to interfaces would involve « artificial » interfaces, i.e. very thin layers matched to both adjacent crystals able to move any interface state out of at least the smaller band gap, very similar to thin graded heterojunctions.

Finally, the difference between low and high injection regimes may be expressed as follows. Though in both cases the reduction of gap surface states is necessary, we must concentrate in the first 
case on the midgap states, mostly originating from defects, and in the second one on near-band-edge states originating from intrinsic distorted bonds. The situations are then different so that the remedies must be different too.

\section{Conclusion.}

The impact of surface and interface recombination processes is bound to increase in the next years, with the progresses in bulk material quality and the decrease of the size of the active regions in devices. However, too few information are still available on the microscopic quantities involved, because both performing and interpreting experiments is at present difficult. It is clear that extended data can only be obtained on surfaces, so that concepts and models must be tested in these cases and then transferred to interfaces. Our first results confirm that the old bulk-like models are refined enough to take into account most of the present data. They also suggest that in most practical cases reducing the surface recombination may be accomplished by replacing the intrinsic states of the surface by those of an « artificial » surface grafted on it. Finally, it may be stressed that the surface recombination may provide a link between electronics which deals with integrated but very sensitive data such as Fermi level pinning, decay times or switching characteristics, and surface physics which gives a deeper insight in microscopic processes.

\section{Acknowledgments.}

The experimental data discussed here are the result of a team work with M. Van Rompay, F. Houzay, F. Barthe and C. Guille, and benefited from many discussions with C. Sébenne.

\section{References}

[1] Stevenson, D. T. and Keyes, R. J., Physica 20 (1954) 1041.

[2] Many, A., Goldstein, Y. and Grover, N. B., in Semiconductor surfaces (Elsevier, New York) 1971.

[3] Aspnes, D. E., Surf. Sci. 132 (1983) 406.

[4] Nagai, H. and Nogushi, Y., J. Appl. Phys. 50 (1979) 1544 ;

Suzuki, T. and Ogawa, M., Appl. Phys. Lett. 34 (1979) 447 ;

Brillson, L. J. and Shapira, Y., Appl. Phys. Lett. 43 (1983) 174.

[5] Moison, J. M., VAN Rompay, M. and Bensoussan, M., Appl. Phys. Lett. 48 (1986) 1362.

[6] Moison, J. M. and Bensoussan, M., Surf. Sci. 168 (1986) 68.
[7] Yablonovitch, E., Allara, D. L., Chang, C. C., GMITTER, T. and Bright, T. B., Phys. Rev. Lett. 57 (1986) 249.

[8] Offsey, S. D., Woodall, J. M., Warren, A. C., KIRChNer, P. D., Chappell, T. I. and PetTit, G. D., Appl. Phys. Lett. 48 (1986) 475.

[9] Guichar, G. M., SÉbenne, C. A. and Garry, G. A., Phys. Rev. Lett. 37 (1976) 1158.

[10] Maigné, P., Ph. D. Thesis, Paris 1986, unpublished.

[11] Moison, J. M., Bensoussan, M. and Houzay, F., Phys. Rev. B 34 (1986) 2018.

[12] Moison, J. M., Van Rompay, M., Guille, C., Houzay, F., BARThe, F. and Bensoussan, M., Proc. of 18th Int. Conf. on the Physics of Semiconductors, Stockholm, 1986. 